\title{
Subdiffraction-Limited Quantum Imaging within a Living Cell
}

\author{
Michael A. Taylor, ${ }^{1,2}$ Jiri Janousek, ${ }^{3}$ Vincent Daria, ${ }^{4}$ Joachim Knittel, ${ }^{1}$ Boris Hage, ${ }^{3,5}$ \\ Hans-A. Bachor, ${ }^{3,4}$ and Warwick P. Bowen ${ }^{2 *}$ \\ ${ }^{1}$ Department of Physics, University of Queensland, St Lucia, Queensland 4072, Australia \\ ${ }^{2}$ Centre for Engineered Quantum Systems, University of Queensland, \\ St Lucia, Queensland 4072, Australia \\ ${ }^{3}$ Department of Quantum Science, Australian National University, Canberra, \\ Australian Capital Territory 0200, Australia \\ ${ }^{4} J o h n$ Curtin School of Medical Research, Australian National University, Canberra, \\ Australian Capital Territory 0200, Australia \\ ${ }^{5}$ Institut für Physik, Universität Rostock, D-18051 Rostock, Germany
}

(Received 21 October 2013; revised manuscript received 16 December 2013; published 4 February 2014)

\begin{abstract}
We report both subdiffraction-limited quantum metrology and quantum-enhanced spatial resolution for the first time in a biological context. Nanoparticles are tracked with quantum-correlated light as they diffuse through an extended region of a living cell in a quantum-enhanced photonic-force microscope. This allows spatial structure within the cell to be mapped at length scales down to $10 \mathrm{~nm}$. Control experiments in water show a $14 \%$ resolution enhancement compared to experiments with coherent light. Our results confirm the long-standing prediction that quantum-correlated light can enhance spatial resolution at the nanoscale and in biology. Combined with state-of-the-art quantum light sources, this technique provides a path towards an order of magnitude improvement in resolution over similar classical imaging techniques.
\end{abstract}

DOI: 10.1103/PhysRevX.4.011017

Subject Areas: Interdisciplinary Physics, Optics, Quantum Physics

The emerging field of quantum imaging utilizes quantum effects to overcome classical imaging constraints. In particular, nonclassical states of light can allow the shotnoise and diffraction limits to be surpassed [1], and quantum-engineered artificial atoms allow new approaches to sensing [2]. The primary motivation for such techniques is in biological imaging [2-6], where any improvement in imaging technology can reveal new levels of cellular complexity. Since subcellular structures often have nanometer-size scales, spatial resolution surpassing the diffraction limit is particularly beneficial. However, neither subdiffraction-limited resolution nor quantum-enhanced resolution have previously been achieved in biology. To date, the only reported demonstration of biological imaging with nonclassical light has been in dispersion compensation for optical coherence tomography [4]. Even in nonbiological demonstrations, both the absolute sensitivity and resolution of optical quantum imaging have been constrained to levels far inferior to state-of-the-art classical technology [5-7]. While unprecedented sensitivity is, in principle, achievable using squeezed states of light $[1,3]$, no method has been experimentally demonstrated capable of utilizing squeezed light in biological imaging.

*wbowen@physics.uq.edu.au

Published by the American Physical Society under the terms of the Creative Commons Attribution 3.0 License. Further distribution of this work must maintain attribution to the author(s) and the published article's title, journal citation, and DOI.
Here, we propose and demonstrate a new quantum imaging method that applies squeezed light in photonic-force microscopy (PFM) [8]. This allows both quantum-enhanced resolution and subdiffraction-limited quantum imaging in biology for the first time, with resolution comparable to leading classical experiments. PFM is a classical subdiffraction-limited imaging technique closely analogous to AFM, but with a nanoscale particle trapped in optical tweezers replacing the probe tip $[8,9]$. As the nanoparticle explores a cell, environmental variations that affect its thermal diffusion can be mapped. PFM has been used to map both 3D surfaces $[10,11]$ and mechanical properties of fluids [12], and has been applied to study cell membranes [8], nanoscale protein motors [13], molecular interactions [14], and, similar to our work here, intracellular viscoelasticity [15]. As is typical of nanoprobe-based microscopy techniques, the spatial resolution achievable in PFM is not constrained by the diffraction limit. The resolution lateral to the motion of the probe particle is constrained by its size. However, similar to AFM, the resolution along the direction of motion is typically limited by measurement signal-to-noise $[10,14]$. Here, we use nonclassical light to improve the signal-to-noise, and thereby demonstrate quantum-enhanced resolution in PFM.

The squeezed-light-enhanced PFM is used to construct one-dimensional profiles of spatial structures within a cell, with features observed at length scales down to $10 \mathrm{~nm}$. Control measurements in water confirm that, for fixed optical power, squeezed light provides $14 \%$ enhancement over the resolution possible with coherent light. A 74\% increase in optical power would be required to achieve this 
level of enhancement without squeezing, increasing the potential for damage $[16,17]$ and photochemical disruption of cellular processes $[17,18]$, which are known to severely limit biological applications of PFM [14]. By demonstrating for the first time that nonclassical light can improve resolution in a biological context, the PFM achieves the key requirement for quantum-enhanced imaging in biology. Our results, further, constitute the first demonstration of quantum-enhanced resolution using squeezed light in any context. When combined with 3D particle tracking, quantum-enhanced nanoscale images of biological structure could be constructed, placing practical applications of quantum imaging with nonclassical light within reach.

The results presented here complement previous quantum imaging experiments using nonclassical light. In imaging applications that simultaneously sample the entire field of view, many spatial modes are captured. Quantum enhancement then requires that quantum correlations are established between a large number of these modes. Such multimode nonclassical light has been applied in proof-of-principle demonstrations of subshot-noise absorption imaging [6], enhanced 2-photon microscopy [19], ghost imaging via photon correlations [7], improved image reconstruction against a noisy background [20], generation of entangled images [21], noiseless image amplification [22,23], and to eliminate unwanted artifacts in optical-coherence tomography [4]. However, practical applications have been precluded by a lack of both bright multimode sources of strongly nonclassical light and high bandwidth array detectors capable of efficiently detecting this light [24]. By contrast, in single-mode or few-modes scenarios, such sources and detectors are readily available [25]. This has enabled quantum-enhanced measurements of spatial parameters such as laser-beam deflection $[3,26]$ and spatial quantum correlations [27]. However, these experiments suffer the apparent major drawback that quantum enhancement is only possible for a number of pixels at most, equal to the number of available single-mode sources of quantum-correlated light. Consequently, they have previously been limited to a maximum of eight pixels [25]. This limitation can be overcome using a scanning probe as demonstrated here for the first time, or an optical raster scan as recently demonstrated in Ref. [5].

In PFM, a probe particle is tracked as it is scanned over the field of view. Variations in measured motion are then studied over a measurement time which is often of the order of minutes [10,11]. Provided the microscope has sufficient stability [14], and that these variations are dominated by static intracellular structure, rather than dynamic cellular processes [28] or nanoscale motion of cellular constituents, this allows a map of the cellular structure to be constructed. The quantum PFM reported in this paper utilizes a recently developed quantum-enhanced nanoparticle tracking technique [29]. While Ref. [29] provided a tool to study temporal fluctuations within living cells, the lack of spatial resolution was a critical shortcoming, preventing any conclusions from being drawn regarding the dominant source of fluctuations in the measured motion, and, therefore, application as a PFM. Here, spatial resolution is introduced and quantum PFM is realized, with thermally driven motion used to scan the probe particle through an extended region of the cell [11].

The experimental setup [shown in Fig. 1(a)] features several important modifications from a conventional PFM, facilitating the use of amplitude-squeezed light to enhance measurement sensitivity. Because most biological processes occur at $\mathrm{Hz}-\mathrm{kHz}$ frequencies, where classical noise sources constrain the possibility of generating squeezing [30], a novel optical lock-in technique [31] is used to evade low-frequency noise and allow quantum enhancement at

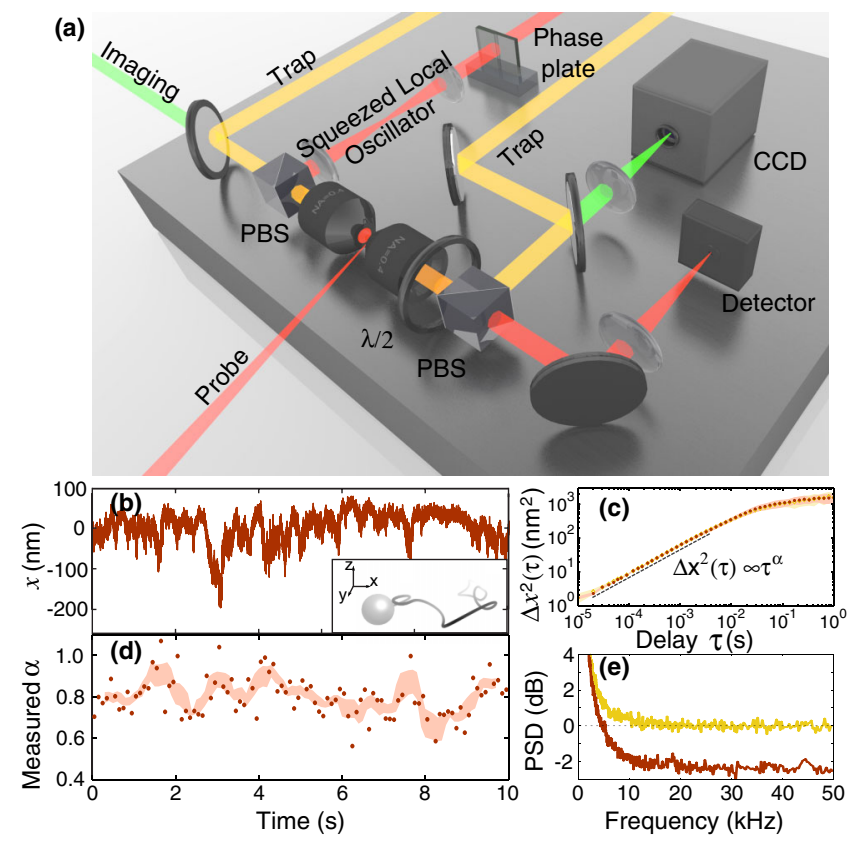

FIG. 1 (color online). Experimental setup. (a) Counterpropagating trapping fields (orange) confine particles between two objectives and are isolated from the detector with polarizing beam splitters (PBS) and wave plates $(\lambda / 2)$. An imaging field (green) allows visual identification of the particles near the optical trap on a CCD camera. The particle tracking measurement relies only on an amplitude-squeezed local oscillator and an amplitude-modulated probe (red), with the probe providing darkfield illumination, and the particle tracking signal arising from interference between scattered light from the probe and the local oscillator. (b) Measured particle motion, which is the $x$ projection of the 3D motion (shown schematically in the inset). (c) The MSD is constructed with both squeezed light (dark red) and coherent light (gold), and $\alpha$ is determined by fitting this to Eq. (2). The classical and squeezed example traces here both yield $\alpha=0.83$. (d) The raw data are divided into $100 \mathrm{~ms}$ segments and the value of $\alpha$ established for each (solid dots). The light red shaded region represents the moving mean and standard error with a 0.5 -s width. (e) The normalized power spectral density (PSD) shows that squeezing suppresses the noise floor by $2.4 \mathrm{~dB}$. 
these frequencies. This is combined with dark-field illumination to remove unwanted light from the measured optical field [32,33]. Additionally, self-homodyne measurement is used, which allows the local oscillator itself to be squeezed, such that the squeezed field perfectly overlaps with the local oscillator at detection even without prior knowledge of the spatial mode shape after propagation through the living cell and high numerical aperture lenses.

Saccharomyces cerevisiae yeast cells are immobilized with an optical trap, and lipid granules of approximately $300 \mathrm{~nm}$ diameter are tracked with either squeezed or coherent light as they diffuse within the cellular cytoplasm. Since the characteristic thermal motion of any particle is determined by the mechanical properties of its surrounding medium, such intracellular particle tracking measurements are commonly used to study the mechanics of cellular cytoplasm [15]. Lipid granules are well suited for use as probe particles within yeast as they occur naturally and can be tracked precisely due to their high refractive index [28,34]. In our experiment, the high-frequency thermal motion of a lipid granule reveals the viscoelastic mechanical properties of the surrounding cytoplasm, while for sufficiently long measurements its slow thermal drift provides spatial resolution by bringing it into contact with different parts of the cellular cytoplasm. Thus, spatial inhomogeneity in the viscoelasticity can be quantified by a single continuous measurement of the lipid granule position. In our experiment, the particle position $x(t)$ transverse from the trap center is measured by combining scattered light from the sample with a local oscillator field that is spatially shaped such that direct measurement of the total power yields the particle position [Fig. 1(b)]. In the same manner as in Ref. [29], mechanical properties of the cytoplasm directly surrounding the nanoparticle could be characterized from its mean-squared displacement (MSD) after a delay $\tau$,

$$
\left\langle\Delta x^{2}(\tau)\right\rangle=\left\langle(x(t)-x(t-\tau))^{2}\right\rangle,
$$

with an example shown in Fig. 1(c). Squeezed light improves the precision by reducing the error with which the MSD can be estimated. This improvement is shown in the measured power spectral density [Fig. 1(e)], with squeezed light lowering the noise floor by $2.4 \mathrm{~dB}$. For short delays, the MSD is dominated by thermal motion and has the form

$$
\left\langle\Delta x^{2}(\tau)\right\rangle=2 D \tau^{\alpha},
$$

where the diffusive parameter $\alpha$ carries information about the mechanical properties of the surrounding medium [35,36]. $\alpha$ is determined for a set of data by fitting the MSD at short delays to Eq. (2). When $\alpha=1$, the motion is diffusive, which is indicative of a random walk type of motion, whereas confinement of the particle causes subdiffusive motion $(0<\alpha<1)$. Subdiffusive motion is an indicator that the cellular cytoplasm exhibits both viscosity and elasticity [35], since to constrict motion the cytoplasm must store mechanical energy. In our experiments, $100 \mathrm{~ms}$ of data is sufficient to precisely determine $\alpha$. Consequently, the measured values of $\alpha$ allow temporal variations in the cellular viscoelasticity to be characterized with $10 \mathrm{~Hz}$ bandwidth.

As lipid particles undergo 3D thermal motion, they are exposed to different parts of the cell [Fig. 1(b) inset]. In a full PFM, 3D motion is tracked through an extended region of the cell. By characterizing the changes in $\alpha$ that occur, it is then possible to construct 3D images of the structure within the cellular cytoplasm [15]. Here, to demonstrate that nonclassical light enables resolution surpassing that possible with coherent light, a proof-of-principle demonstration is achieved using $1 \mathrm{D}$ particle tracking along the $x$ axis, with the coordinates $y$ and $z$ not determined. This allows 1D profiles of $\alpha(x)$ to be constructed following the projection of the trajectory onto the $x$ axis.

A series of experiments is performed in which the motion of lipid particles are tracked with quantum-enhanced precision for $10 \mathrm{~s}$ as they diffused through the cell. The data from each experiment are separated into 100-ms segments, with both $\alpha$ and the mean position along the $x$ axis determined for each segment. As the particle diffuses, a profile of $\alpha$ is generated as a function of $x$, with four representative profiles shown in Fig. 2. As can be seen, the particles explore a range of approximately $120 \mathrm{~nm}$ along the $x$ axis over the 10-s measurement interval, consistent with the MSD in Fig. 1(c) extrapolated to longer delays. The directly obtained data exhibit substantial noise both from the measurement process and due to the unknown trajectory of the particle in the $y$ and $z$ directions. To identify statistically resolvable features, the running mean and standard error of $\alpha$ are calculated along the $x$ axis, with a 10-nm averaging window defining the spatial resolution. Since increased spatial averaging makes small changes in $\alpha$ easier to resolve, an intrinsic compromise is present between spatial resolution and contrast, with the latter defined as the statistical uncertainty in $\alpha$. The choice of $10-\mathrm{nm}$ spatial resolution is found to provide sufficient contrast to observe cellular structure.

The observed spatial structure varies between measurements of $\alpha(x)$ because the particle follows different 3D trajectories. Gradual linear changes in $\alpha$ are observed [e.g., Fig. 2(a)] that suggest a spatial gradient in the molecular crowding along the $x$ axis [37], along with narrow dips in $\alpha$ [e.g., Fig. 2(b) at $40 \mathrm{~nm}$ ], suggestive of barriers in the cytoplasm, areas of homogeneity [e.g., Fig. 2(c)], and peaks in $\alpha$ [e.g., Fig. 2(d) at $-55 \mathrm{~nm}$ ], which may follow from small voids in the cytoplasmic structure. Since only the projection of the particle motion onto the $x$ axis is tracked, it is not possible to define the complete trajectory along which these 1D profiles are taken. This obscures the biological origin of observed features. For instance, the narrow dip in $\alpha$ seen in Fig. 2(b) could result from a range of subcellular components including an actin filament or the edge of a larger organelle, even though these have markedly different $3 \mathrm{D}$ profiles. The $3 \mathrm{D}$ motion of the 

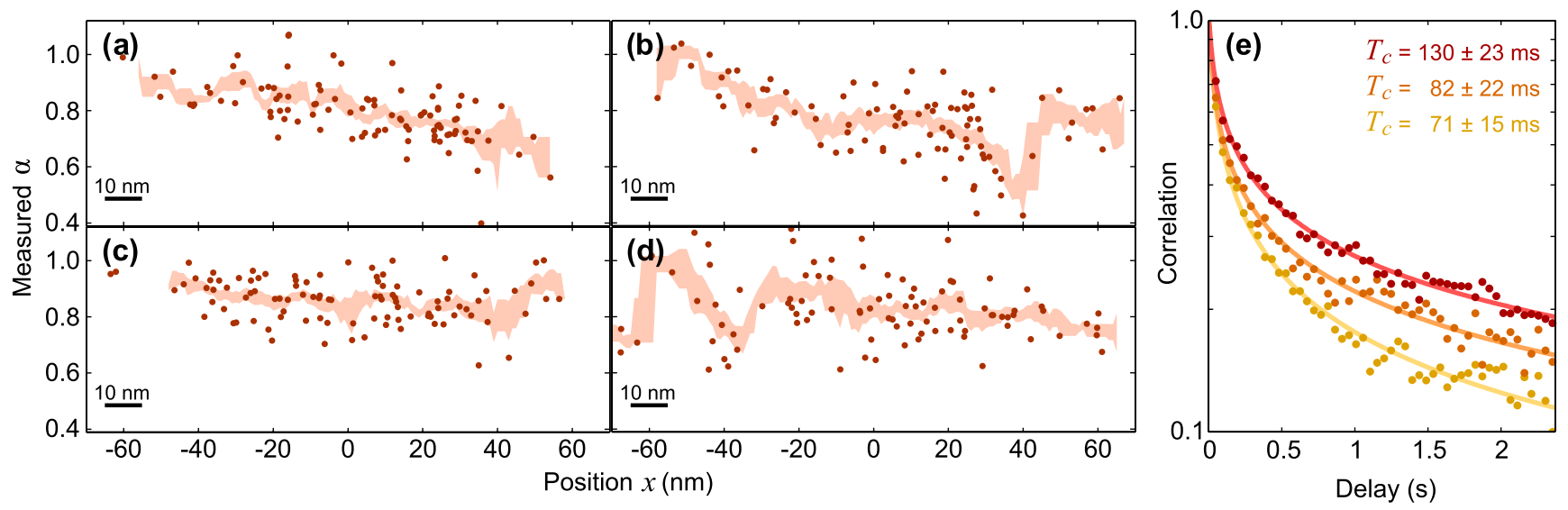

FIG. 2 (color online). 1D profiles of $\alpha$. Each circle represents a single measurement of $\alpha$ versus $x$ using a 100-ms set of data. The shaded regions represent the running mean and standard error with 10-nm resolution (thick black bar). Each profile is recorded minutes apart to allow the particle time to diffuse to different regions of the cell with qualitatively different spatial structures. The particle confinement is greatest where $\alpha$ is lowest, such as the dip about $40 \mathrm{~nm}$ in (b), and the particle movement is most free when $\alpha$ is highest, such as the peak at $-55 \mathrm{~nm}$ in (d). To verify that the changes in $\alpha$ are spatial, correlations between sequential measurements of $\alpha(x)$ are analyzed for three sets of data in (e). The circles are experimentally determined correlations between a series of measurements, which are well fitted by the predicted relation (lines) explained in the Supplemental Material, Sec. S1 [38]. By fitting data to this theory, the characteristic time $T_{c}$ for the particle to diffuse into uncorrelated regions of the cell can be determined. Note that the decay in correlation restricts the duration over which $\alpha(x)$ profiles can be constructed. Although the characteristic times found here are in the range of $0.1 \mathrm{~s}$, correlations are found to persist for sufficient time to construct the 10-s $\alpha(x)$ profiles shown here.

particle also degrades the contrast of narrow features by averaging measurements of $\alpha$ from a range of positions along the $y$ and $z$ axes. These limitations could be resolved by incorporating our technique in a 3D PFM [8] that maps the complete trajectory of the particle. It may then be possible to generate a quantum-enhanced 3D image of the cell, with quantum enhancement required only for one axis from which $\alpha$ could be determined.

In addition to this technological improvement, it will also be important to minimize nonideal effects in future quantum PFM systems. It is particularly important that the effect of background-scattering centers on the measured signal be characterized, and unwanted scattered light eliminated as much as possible (see Supplemental Material, Sec. S3 [38]). This technical difficulty has already been addressed in previous classical measurements of anomalous diffusion within cells [34]. While it requires careful attention, it does not present a fundamental barrier to use of this quantum imaging technique in practical biological experiments.

Importantly, even though the biological origin of the profiles in Figs. 2(a)-2(d) is obscured, the measured changes in $\alpha$ can be rigorously shown to originate from the spatial structure within the cell. In a static spatially varying environment, spatial correlations between $\alpha(x)$ profiles should decay exponentially with the time between the profiles, as the unknown motion along the $y$ and $z$ axes brings the particle into different regions of the cell. By contrast, temporal changes in the cell [28] or drifts in the apparatus which produce fluctuations in $\alpha$ should not exhibit any correlations between profiles. A sequence of $\alpha(x)$ profiles are measured at a rate of $20 \mathrm{~s}^{-1}$, and correlations between the profiles are calculated. The experimental data [Fig. 2(e)] show excellent agreement with the predicted correlation decay for measurement in a static spatially varying environment (see Supplemental Material, Sec. S1 [38]), thus confirming that the $\alpha$ profiles reflect spatial structure rather than temporal fluctuations. Furthermore, this analysis of correlations allows the local length scale of viscoelastic structure to be determined in the region of the nanoparticle. This length scale can be found by combining the MSD measured in Fig. 1(c) with the fitted characteristic time $T_{c}$ over which the particle diffuses into an uncorrelated region of the cell (see Supplemental Material, Sec. S1 [38]). The length scales of the viscoelastic structure in three different regions are determined to be $46.9 \pm 1.3,43.7 \pm 2.4$, and $42.6 \pm 1.9 \mathrm{~nm}$ [Fig. 2(e)] demonstrating that changes in the characteristic length of the spatial structure in different parts of the cell can be statistically distinguished with nanometer precision.

Because of the complexity of the intracellular environment, the quantum resolution enhancement achieved in the PFM was characterized via control experiments on 1- $\mu \mathrm{m}-$ radius silica beads in water, rather than directly in vivo. This approach of using simple well-understood control experiments is standard when calibrating resolution in PFM $[8,10,14] . \alpha(x)$ profiles are constructed from $80 \mathrm{~s}$ of data using both squeezed and coherent light. In this case, $2.5 \mathrm{~dB}$ of squeezing is measured, which closely approaches the enhancement achieved in biological measurements. These measurements show no statistically significant spatial structure (Fig. 3), with $\alpha=1$ at all spatial locations, as expected for Brownian motion. This lack of statistically 

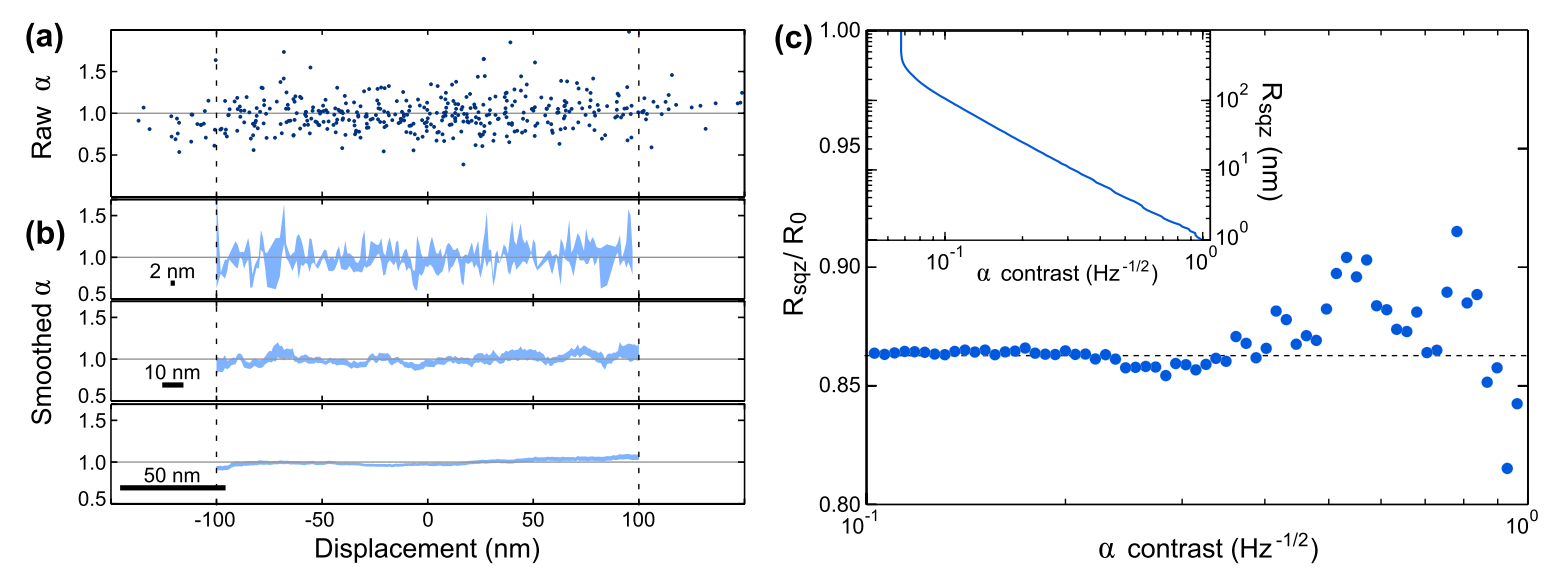

FIG. 3 (color online). Characterization of the spatial resolution. To calibrate the resolution enhancement achieved here, a profile of $\alpha(x)$ is constructed by tracking particles in water. The individual measurements are shown in (a), while (b) shows the corresponding moving mean and standard error calculated over a 200-nm range with spatial resolution of 2, 10, and $50 \mathrm{~nm}$. The data closely follow the expected $\alpha=1$ result (horizontal line). (c), The ratio of spatial resolutions with squeezed and coherent light is shown as a function of contrast in $\alpha$. The $\alpha$ contrast values shown are normalized into units of $\mathrm{Hz}^{-1 / 2}$ to account for the improvement in absolute contrast as data are accumulated. For a fixed contrast, the spatial resolution achievable with squeezing $\left(R_{\text {sqz }}\right)$ is improved by approximately $14 \%$ when compared to coherent light $\left(R_{0}\right)$. The absolute spatial resolution achievable using squeezed light is plotted in the inset. Since the number of points being averaged is proportional to the spatial resolution, the sensitivity scales as the inverse square root of spatial resolution until the averaging window width becomes comparable to the spatial range of the measured data.

significant variation provides further verification that the structure observed in vivo can be attributed to changes in $\alpha$, rather than to drifts in the apparatus. Since water is homogeneous with $\alpha=1$ throughout, the variation in this data allows the statistical uncertainty, or contrast, of our measurements of $\alpha$ to be determined. The $\alpha$ contrast is characterized as a function of spatial resolution by varying the width of the running average along $x$, as shown representatively in Fig. 3(b). As the width increases, the spatial resolution is degraded, but the contrast in $\alpha$ improves since more data are averaged at each position along $x$ [see Fig. 3(c) inset]. By comparing the resolution required to achieve a fixed contrast with and without squeezed light, it is possible to confirm for the first time that squeezed light can be used to enhance spatial resolution. Furthermore, this also provides the first demonstration of quantum-enhanced spatial resolution in a biological context. As shown in Fig. 3(c), in this proof-of-principle experiment, squeezed light allows a $14 \%$ improvement in resolution for contrasts in $\alpha$ ranging from 0.1 to $1 \mathrm{~Hz}^{-1 / 2}$.

To take a specific example, the biological profiles shown in Fig. 2 use $10-\mathrm{nm}$ resolution and include $10 \mathrm{~s}$ of accumulated data. In our control experiments in water, this resolution and accumulation time would allow structures that alter $\alpha$ by 0.1 to be resolved [see Fig. 3(c) inset]. In comparison, a resolution of $12 \mathrm{~nm}$ would be required to resolve such features with coherent light. It is important to note that both the measurement sensitivity and the spatial range of diffusion differ between this calibration with silica beads and the measurements in biology. Consequently, the $\alpha$ contrast determined here differs from that achieved in vivo, where the average standard error in $\alpha$ is 0.04 .
Importantly, the quantum resolution enhancement is independent of the absolute level of contrast, as can be seen over an order of magnitude in Fig. 3(c). Therefore, even though the absolute resolution differs between in vivo and control experiments, the quantum resolution enhancement predicted here can be expected to accurately represent the in vivo enhancement.

In absolute terms, the resolution achieved here is comparable to that of leading classical PFM measurements of viscoelasticity $[13,15]$. Furthermore, the resolution could be substantially improved using an increased level of squeezing. With $10 \mathrm{~dB}$ of measured squeezing, as reported in a number of experiments [39,40], an order of magnitude enhancement should be feasible (see Supplemental Material, Sec. S2 [38]). This could potentially allow angstrom-level resolution. In principle, further enhancement may be possible by using more sophisticated quantum measurements [41,42], with recent theoretical results predicting that an array of photonnumber-resolving detectors could even allow particle tracking at the de Broglie limit [42].

When combined with the advances described above, the technology introduced here could help to answer important questions related to the nanoscale structure within cells. It has potential that extends beyond mapping of organelle positions, since thermal motion is critical to the operation of the cell and mediates important functions such as chemical reactions [43] and protein folding [44]. It has been shown that the optimal diffusive regime is different for storage, transport, and chemical reactions [43], and that in some regions of the cell, structures that influence diffusion are organized at the nanoscale [45]. It remains unknown to what extent these nanoscale structural variations reflect an 
underlying biological function. We anticipate that in the future quantum imaging could play an important role in answering such questions.

Our results complement recent biological applications of quantum-engineered diamond probes with nitrogen vacancy (NV) centers, which have enabled thermal [46] and magnetic cellular imaging [47-49]. None of these applications have achieved subdiffraction-limited resolution, as they rely on optically resolvable arrays of stationary NV probes [46-49], confining them to the study of relatively large cellular structures and organelles. The resolution achieved here is over an order of magnitude finer, providing the possibility to observe important nanoscale cellular structures such as membranes, actin networks, and individual proteins. Since the approach is, in principle, transferable to NV nanodiamond-based imaging, it could also open the door to simultaneous subdiffraction-limited imaging of structure, temperature, and magnetic fields.

In summary, we report the first application of quantum imaging techniques to subdiffraction-limited biological imaging and demonstrate that nonclassical light can improve spatial resolution in biological applications. The viscoelastic structure within a living yeast cell is sampled along the trajectory of a thermally driven nanoparticle, revealing spatial structure with length scales down to $10 \mathrm{~nm}$. Control experiments in water show that the spatial resolution is enhanced by $14 \%$ through the use of squeezed light. Future experiments that apply this quantum-enhanced photonic-force microscope with improved technology may enable resolution of a sub-nm structure in vivo.

This work was supported by the Australian Research Council Discovery Project Contract No. DP0985078 and the Australian Research Council Centre of Excellence for Engineered Quantum Systems Contract No. CE110001013.

[1] M. I. Kolobov and C. Fabre, Quantum Limits on Optical Resolution, Phys. Rev. Lett. 85, 3789 (2000).

[2] L.P. McGuinness et al., Quantum Measurement and Orientation Tracking of Fluorescent Nanodiamonds inside Living Cells, Nat. Nanotechnol. 6, 358 (2011).

[3] N. Treps, U. Andersen, B. Buchler, P. K. Lam, A. Maître, H.-A. Bachor, and C. Fabre, Surpassing the Standard Quantum Limit for Optical Imaging Using Nonclassical Multimode Light, Phys. Rev. Lett. 88, 203601 (2002).

[4] M. B. Nasr, D. P. Goode, N. Nguyen, G. Rong, L. Yang, B. M. Reinhard, B. E. A. Saleh, and M. C. Teich, Quantum Optical Coherence Tomography of a Biological Sample, Opt. Commun. 282, 1154 (2009).

[5] T. Ono, R. Okamoto, and S. Takeuchi, An EntanglementEnhanced Microscope, Nat. Commun. 4, 2426 (2013).

[6] G. Brida, M. Genovese, and I. Ruo Berchera, Experimental Realization of Sub-Shot-Noise Quantum Imaging, Nat. Photonics 4, 227 (2010).
[7] T. B. Pittman, Y.H. Shih, D. V. Strekalov, and A. V. Sergienko, Optical Imaging by Means of Two-Photon Quantum Entanglement, Phys. Rev. A 52, R3429 (1995).

[8] E.-L. Florin, A. Pralle, J. K. H. Hörber, and E. H. K. Stelzer, Photonic-Force Microscope Based on Optical Tweezers and Two-Photon Excitation for Biological Applications, J. Struct. Biol. 119, 202 (1997).

[9] L. P. Ghislain and W. W. Webb, Scanning-Force Microscope Based on an Optical Trap, Opt. Lett. 18, 1678 (1993).

[10] M. E. J. Friese, A. G. Truscott, H. Rubinsztein-Dunlop, and N. R. Heckenberg, Three-Dimensional Imaging with Optical Tweezers, Appl. Opt. 38, 6597 (1999).

[11] C. Tischer, S. Altmann, S. Fišinger, J. K. H. Hörber, E. H. K. Stelzer, and E.-L. Florin, Three-Dimensional Thermal Noise Imaging, Appl. Phys. Lett. 79, 3878 (2001).

[12] A. Pralle, E.-L. Florin, E. H. K. Stelzer, and J. K. H. Hörber, Local Viscosity Probed by Photonic-Force Microscopy, Appl. Phys. A 66, S71 (1998).

[13] T. Scholz, S. M. Altmann, M. Antognozzi, C. Tischer, J. K. H. Hörber, and B. Brenner, Mechanical Properties of Single Myosin Molecules Probed with the PhotonicForce Microscope, Biophys. J. 88, 360 (2005).

[14] A. Rohrbach, C. Tischer, D. Neumayer, E. L. Florin, and E. H. Stelzer, Trapping and Tracking a Local Probe with a Photonic-Force Microscope, Rev. Sci. Instrum. 75, 2197 (2004).

[15] E. Bertseva et al., Intracellular Nanomanipulation by a Photonic-Force Microscope with Real-Time Acquisition of a 3D Stiffness Matrix, Nanotechnology 20, 285709 (2009).

[16] E. J. G. Peterman, F. Gittes, and C. F. Schmidt, LaserInduced Heating in Optical Traps, Biophys. J. 84, 1308 (2003).

[17] K. C. Neuman, E. H. Chadd, G. F. Liou, K. Bergman, and S. M. Block, Characterization of Photodamage to Escherichia coli in Optical Traps, Biophys. J. 77, 2856 (1999).

[18] R. Lubart, R. Lavi, H. Friedmann, and S. Rochkind, Photochemistry and Photobiology of Light Absorption by Living Cells, Photomed. Laser Surg. 24, 179 (2006).

[19] H.-B. Fei, B. M. Jost, S. Popescu, B. E. A. Saleh, and M. C. Teich, Entanglement-Induced Two-Photon Transparency, Phys. Rev. Lett. 78, 1679 (1997).

[20] E. D. Lopaeva, I. R. Berchera, I. P. Degiovanni, S. Olivares, G. Brida, and M. Genovese, Experimental Realization of Quantum Illumination, Phys. Rev. Lett. 110, 153603 (2013).

[21] V. Boyer, A. M. Marino, R. C. Pooser, and P. D. Lett, Entangled Images from Four-Wave Mixing, Science 321, 544 (2008).

[22] A. Mosset, F. Devaux, and E. Lantz, Spatially Noiseless Optical Amplification of Images, Phys. Rev. Lett. 94, 223603 (2005).

[23] L. Lopez, N. Treps, B. Chalopin, C. Fabre, and A. Maître, Quantum Processing of Images by Continuous Wave Optical Parametric Amplification, Phys. Rev. Lett. 100, 013604 (2008).

[24] M. Lassen, V. Delaubert, J. Janousek, K. Wagner, H.-A. Bachor, P. K. Lam, N. Treps, P. Buchhave, C. Fabre, and C. C. Harb, Tools for Multimode Quantum Information: Modulation, Detection, and Spatial Quantum Correlations, Phys. Rev. Lett. 98, 083602 (2007). 
[25] S. Armstrong, J.-F. Morizur, J. Janousek, B. Hage, N. Treps, P. K. Lam, and H.-A. Bachor, Programmable Multimode Quantum Networks, Nat. Commun. 3, 1026 (2012).

[26] N. Treps, N. Grosse, W. P. Bowen, C. Fabre, H.-A. Bachor, and P. K. Lam, A Quantum Laser Pointer, Science 301, 940 (2003).

[27] J. Janousek, K. Wagner, J. F. Morizur, N. Treps, P. K. Lam, C. C. Harb, and H.-A. Bachor, Optical Entanglement of Copropagating Modes, Nat. Photonics 3, 399 (2009).

[28] C. Selhuber-Unkel, P. Yde, K. Berg-Sørensen, and L. B. K. Oddershede, Variety in Intracellular Diffusion during the Cell Cycle, Phys. Biol. 6, 025015 (2009).

[29] M. A. Taylor, J. Janousek, V. Daria, J. Knittel, B. Hage, H.-A. Bachor, and W. P. Bowen, Biological Measurement beyond the Quantum Limit, Nat. Photonics 7, 229 (2013).

[30] K. McKenzie, N. Grosse, W. P. Bowen, S. E. Whitcomb, M. B. Gray, D. E. McClelland, and P. K. Lam, Squeezing in the Audio Gravitational-Wave Detection Band, Phys. Rev. Lett. 93, 161105 (2004).

[31] M. A. Taylor, J. Knittel, and W. P. Bowen, Optical Lock-In Particle Tracking in Optical Tweezers, Opt. Express 21, 8018 (2013).

[32] S. Kudo, Y. Magariyama, and S. I. Aizawa, Abrupt Changes in Flagellar Rotation Observed by Laser Dark-Field Nicroscopy, Nature (London) 346677 (1990).

[33] M. A. Taylor and W. P. Bowen, Enhanced Sensitivity in Dark-Field Microscopy by Optimizing the Illumination Angle, Appl. Opt. 52, 5718 (2013).

[34] I. M. Tolić-Nørrelykke, E.-L. Munteanu, G. Thon, L. Oddershede, and K. Berg-Sørensen, Anomalous Diffusion in Living Yeast Cells., Phys. Rev. Lett. 93, 078102 (2004).

[35] F. Gittes, B. Schnurr, P. D. Olmsted, F. C. MacKintosh, and C. F. Schmidt, Microscopic Viscoelasticity: Shear Moduli of Soft Materials Determined from Thermal Fluctuations, Phys. Rev. Lett. 79, 3286 (1997).

[36] T. G. Mason, K. Ganesan, J. H. Van Zanten, D. Wirtz, and S. C. Kuo, Particle Tracking Microrheology of Complex Fluids, Phys. Rev. Lett. 79, 3282 (1997).

[37] M. Weiss, M. Elsner, F. Kartberg, and T. Nilsson, Anomalous Subdiffusion is a Measure for Cytoplasmic Crowding in Living Cells, Biophys. J. 87, 3518 (2004).

[38] See Supplemental Material at http://link.aps.org/ supplemental/10.1103/PhysRevX.4.011017 for the models used to predict image correlations and the achievable quantum enhancement, and for a discussion of background scattering.

[39] M. S. Stefszky, C. M. Mow-Lowry, S. S. Y. Chua, D. A. Shaddock, B. C. Buchler, H. Vahlbruch, A. Khalaidovski, R. Schnabel, P. K. Lam, and D. E. McClelland, Balanced Homodyne Detection of Optical Quantum States at Audio-Band Frequencies and Below, Classical Quantum Gravity 29, 145015 (2012).

[40] M. Mehmet, H. Vahlbruch, N. Lastzka, K. Danzmann, and R. Schnabel, Observation of Squeezed States with Strong Photon-Number Oscillations, Phys. Rev. A 81, 013814 (2010).

[41] M. A. Taylor, J. Knittel, and W. P. Bowen, Fundamental Constraints on Particle Tracking with Optical Tweezers, New J. Phys. 15, 023018 (2013).

[42] M. Tsang, Quantum Imaging beyond the Diffraction Limit by Optical Centroid Measurements, Phys. Rev. Lett. 102, 253601 (2009).

[43] G. Guigas and M. Weiss, Sampling the Cell with Anomalous Diffusion-The Discovery of Slowness, Biophys. J. 94, 90 (2008).

[44] H. Frauenfelder, P. W. Fenimore, G. Chen, and B. H. McMahon, Protein Folding is Slaved to Solvent Motions, Proc. Natl. Acad. Sci. U.S.A. 103, 15469 (2006).

[45] Y. Tseng, J. S. Lee, T. P. Kole, I. Jiang, and D. Wirtz, Microorganization and Viscoelasticity of the Interphase Nucleus Revealed by Particle Nanotracking, J. Cell Sci. 117, 2159 (2004).

[46] G. Kucsko, P. C. Maurer, N. Y. Yao, M. Kubo, H. J. Noh, P. K. Lo, H. Park, and M. D. Lukin, Nanometre-Scale Thermometry in a Living Cell, Nature (London) 500, 54 (2013).

[47] D. Le Sage, K. Arai, D. R. Glenn, S. J. DeVience, L. M. Pham, L. Rahn-Lee, M. D. Lukin, A. Yacoby, A. Komeili, and R. L. Walsworth, Optical Magnetic Imaging of Living Cells, Nature (London) 496, 486 (2013).

[48] S. Steinert, F. Ziem, L. T. Hall, A. Zappe, M. Schweikert, N. Götz, A. Aird, G. Balasubramanian, L. Hollenberg, and J. Wrachtrup, Magnetic Spin Imaging under Ambient Conditions with Subcellular Resolution, Nat. Commun. 4, 1607 (2013).

[49] S. Kaufmann et al., Detection of Atomic Spin Labels in a Lipid Bilayer Using a Single-Spin Nanodiamond Probe, Proc. Natl. Acad. Sci. U.S.A. 110, 10894 (2013). 